\title{
Arbor
}

\section{La Aviación Militar Española: una historia corta pero de gran intensidad}

José Sánchez Méndez

Arbor CLXXI, 674 (Febrero 2002), 187-216 pp.

En este artículo, su autor hace un recorrido histórico, excelentemente documentado, por toda la historia de la Aviación Militar Española, desde sus inicios, allá por 1910, hasta nuestros días.

Como puntos más importantes podemos destacar:

- La Campaña de Marruecos, donde se realizó el primer bombardeo específicamente aéreo y se constato la necesidad de contar con apoyo aéreo para el desarrollo de las operaciones militares.

- Los grandes vuelos de la Aviación Militar Española: Palos de la Frontera-Buenos Aires, Madrid-Manila, Melilla-Santa Isabel.

- La época de la guerra civil española, escenario de las nuevas doctrinas aéreas y de ensayo de nuevas tácticas que marcarían la futura actuación de la Aviación en la II Guerra Mundial.

- La creación del Ejército del Aire, la "Escuadrilla Azul" y el difícil quinquenio 1945-50, hasta que se inició su modernización.

- En el área operativa se trata la actuación del Poder Aéreo en Ifni, Sahara y los Balcanes.

- En el área de organización se habla sobre el programa ORGEA (Organización del Ejército del Aire), la creación del Ministerio de Defensa y la estructura orgánica actual.

- En lo referente a material, destacan la creación de la red de alerta y control, la llegada de los primeros cazas, para terminar con los más recientes planes de modernización.

- Por último, debemos hacer mención especial de nuestra integración en Europa e ingreso en la OTAN, por la gran influencia que estos dos hechos han tenido y tendrán en el futuro del Ejército del Aire. 


\section{Introducción}

Los antecedentes del Ejército del Aire tienen lugar en el primer tercio del Siglo XX, con el nacimiento, desarrollo y brillantísima actuación de la Aeronáutica Militar Española durante los treinta años anteriores a 1939.

Sería el eco del primer vuelo de los hermanos Wright lo que llevaría a que un pequeño grupo de ingenieros militares españoles, todos ellos pilotos de globos y dirigibles, quisieran conocer de cerca los primeros vuelos con aeroplanos que ambos hermanos realizaban en Europa y los de otros pioneros de la Aviación de nuestro Viejo Continente. Al frente de dichos ingenieros se encontraba el teniente coronel Pedro Vives Vich quien estaba considerado como el impulsor de la Aerostación en nuestra Patria.

El 2 de abril de 1910 una Real Orden creó los Servicios de Aerostación, Aeronáutica y Aviación dependientes del Ejército y uno de sus cometidos era estudiar la adquisición del tipo de aeroplano que fuese más conveniente para nuestras necesidades. Pocos meses después se construyó en Madrid el primer aeródromo, en unos terrenos próximos a la carretera de Extremadura, junto a la Venta del Clares y al que se le dió el nombre de Cuatro Vientos. El primer español que obtuvo el título de piloto aviador fue Benito Loygorri y se le otorgó el 20 de agosto de 1910 tras unos vuelos realizados en Francia y el siguiente sería el Infante de Orleáns, el 23 de octubre del mismo año, con lo que se convirtió en el primer aviador militar de nuestro país. Con el fin de formar en España a nuestros pilotos de aeroplanos, en febrero de 1911 se creaba una Escuela de Aviación, cuyos primeros alumnos fueron los capitanes Alfredo Kindelán, Emilio Herrera y Enrique Arrillaga y los tenientes Eduardo Barrón y José Ortiz de Echagüe, siendo designado Kindelán como responsable del aeródromo.

La necesidad de adecuar las adquisiciones de nuevos aeroplanos y el creciente número de aviadores, llevaría al Gobierno a adaptar la organización creada en 1910 a la realidad que había en aquel momento. Así, el 28 de febrero de 1913 por Real Decreto se constituía el Servicio de Aeronáutica Militar que comprendía las ramas de Aerostación y de Aviación, siendo nombrado como jefe del mismo el ya coronel Vives y de las ramas de Aerostación y de Aviación el comandante Antonio Cue Vidaña y el capitán Kindelán respectivamente.

\section{La Aviación Militar Española en la campaña de Marruecos}

La responsabilidad de España en el ejercicio del Protectorado en la zona norte de Marruecos, que le había sido confiada como consecuencia 


\section{La Aviación Militar Española}

del Tratado de 27 de noviembre de 1912 suscrito con Francia, obligó a que fuerzas militares españolas tuvieran que iniciar a principios de 1913 una serie de acciones a fin de someter a las cábilas que no reconocían la autoridad del Sultán. Para estas acciones contaban con una unidad del Servicio de Aerostación del Ejército. La creación, ya mencionada, del Servicio de Aeronáutica Militar en febrero de 1913, permitió el envío a Marruecos de una escuadrilla de doce aeroplanos al mando del capitán Kindelán, que llegaría a Tetuán, capital del Protectorado, en octubre de dicho año. Dos meses más tarde, el 17 de diciembre, un biplano Lohner, tripulado por los capitanes Barrón y Cifuentes realizó el primer bombardeo específicamente aéreo de la historia aeronáutica mundial, al utilizar para ello bombas de diseño especial para aviación y un rudimentario visor de fabricación austriaca.

Durante la I Guerra Mundial el número de escuadrillas se elevó a tres, que se estacionaban, además de Tetuán, en dos nuevos aeródromos, Melilla y Larache y su actuación consistía en apoyar a las operaciones de pacificación y limpieza, aunque su intensidad se redujo al mínimo para evitar cualquier incidente que pudiera poner en peligro la neutralidad española. Pero terminado el conflicto europeo y compaginando la acción militar con la política, las operaciones que hasta enero de 1920 se habían limitado a la zona occidental se extendieron también a la oriental, donde el nuevo comandante general de Melilla, general Fernández Silvestre había comenzado una arriesgada campaña acompañada de un éxito quizás excesivo y que le llevaría a alcanzar la Bahía de Alhucemas, donde se encontraba la cábila del caudillo de la rebelión, Sidi Mohamed Abdel-Krim El Jatabi.

En junio de 1921 sería asaltada por las harkas rifeñas la posición de Abarrán y al mes siguiente la Igueriben, sin que el general Silvestre hubiera podido socorrerlas desde Annual, a pesar del apoyo aéreo de la escuadrilla que se había constituído anteriormente en Melilla. En un clima de desmoralización se ordenó la retirada y en ella murieron miles de soldados y el propio general Silvestre.

El 24 de julio caía el aeródromo de Melilla, sucumbiendo así la zona de la Comandancia General, improvisándose en los terrenos de la Hípica de la ciudad un campo de aviación desde el que comenzaron a actuar cinco aeroplanos biplaza de bombardeo, que comenzaron a abastecer desde el aire a las fuerzas sitiadas en Monte Arruit, pero esta posición cayó el 9 de agosto pereciendo más de dos mil hombres y siendo hecho prisionero el general Navarro y varios centenares de soldados. La tragedia produjo una profunda conmoción en la opinión pública nacional, que daría paso a una enérgica reacción de los españoles dispuestos a restaurar el 
prestigio de la nación. Así, una suscripción popular permitió la rápida adquisición de 50 aeroplanos, sumándose otro elevado número fabricados en España por la Hispano Aviación.

Iniciada por el Ejército una gran ofensiva conocida como Campaña de Desquite, en septiembre se recuperaron el aeródromo de Melilla y Monte Arruit, protegiendo la Aviación estos avances. Poco después, en noviembre se creaban las Fuerzas Aéreas de Marruecos que contaban con seis escuadrillas y cuyo mando fue confiado al coronel de Ingenieros Jorge Soriano, lo que facilitó las operaciones terrestres que permitirían recuperar el terreno perdido en 1921 y llevar cierta calma al territorio del Protectorado.

En 1923 Abd-el-Krim se proclamaba sultán de Marruecos y en el mes de julio inició una serie de ataques contra las posiciones españolas que comenzó por Tizi Aza, que quedó sitiada. La decidida intervención de las Fuerzas Aéreas, que atacaban a muy baja altura a las harkas rifeñas, facilitó la llegada de una columna terrestre que liberaría a la posición. La forma de volar de nuestros aviadores sería denominada en toda Europa «vuelo a la española», puesto que atacaban sin descanso para abastecer a las posiciones sitiadas y entregaban el agua en sacos conteniendo barras de hielo y lo hacían a tan baja altura que recibían numerosos impactos de bala de arriba hacia abajo. Días después tendrían que repetir su actuación para permitir la liberación de Tifarauín y posteriormente y con gran frecuencia se vieron obligados a volar prácticamente sobre casi todo el territorio en apoyo de las fuerzas terrestres.

La ofensiva generalizada de Abd-el-Krim iniciada en abril de 1925 contra la zona sur del Protectorado bajo administración francesa, llevó a la rotura del frente y a la caída y aniquilamiento de numerosas guarniciones del Ejército francés, que causó un desastre de proporciones muy superiores al de España en Annual. El prestigioso mariscal Petain, al ser nombrado nuevo Comandante General del Protectorado francés, decidió entrevistarse con el entonces jefe del Gobierno español, general Primo de Rivera, reuniéndose ambos militares en Algeciras, para estudiar la situación marroquí, acordando los dos un plan de ataque contra Abd-el-Krim, que incluía el desembarco de un fuerte contingente español en la Bahía de Alhucemas.

La entidad del contingente español fue de veinte mil hombres, dividido en dos brigadas de idénticos efectivos, una procedente de Ceuta y otra de Melilla, fijándose en el plan de operaciones como día $\mathrm{D}$ y hora $\mathrm{H}$ el 7 de septiembre a las 04,00 horas. La fuerza terrestre fue transportada por mar protegida por dos potentes escuadras navales, siendo reforzada la procedente de Melilla con varios buques de guerra franceses. Pero para poder garantizar el éxito y a la vista del fracaso aliado en Gallípoli durante la I Guerra Mundial, se consideró necesario e imprescindible dis- 


\section{La Aviación Militar Española}

poner de un fuerte apoyo aéreo. La cantidad de aviones que se concentró en el Norte de África se elevó a 162, de los cuales 136 pertenecían a la Aeronáutica Militar, 18 hidroaviones procedían de la Aeronáutica Naval, 6 bombarderos de la Aeronáutica Militar francesa y dos hidroaviones ambulancia cedidos por la Cruz Roja española.

Durante los 24 días de operaciones la actuación de la Aviación Militar española fue decisiva para el éxito del desembarco y posterior ocupación del Cuartel General de Abd-el-Krim, a pesar de que durante varios días las adversas circunstancias meteorológicas impidieron o dificultaron el vuelo de los aeroplanos. Los aviadores españoles volaron 1462 horas y lanzaron 136 toneladas de explosivos, siendo la duración media de cada vuelo de 2 horas. Solamente seis aviadores resultaron heridos, perdiéndose ocho aviones y otros siete sufrieron diversos daños. De esta forma se iniciaría la total pacificación del territorio que finalizaría en 1927.

La Aviación Militar española fue un instrumento esencial y decisivo en la Campaña de Marruecos y sin la cual hubiera sido imposible la pacificación del Protectorado, si bien tuvo que pagar un alto precio por ello. Sirva de muestra que desde el 21 de julio de 1921, fecha en la que comenzó la retirada de Annual, hasta el 10 de julio de 1927 en la que concluyó la Campaña las bajas fueron las siguientes:

31 aviadores murieron en combate por el fuego enemigo.

38 aviadores resultaron muertos en accidentes aéreos por muy diversas causas, disparos de los rifeños, mal tiempo o falta de combustible.

36 aviadores fueron gravemente heridos.

4 serían hechos prisioneros.

65 aviones fueron derribados.

Por otra parte, de las 17 Cruces Laureadas de San Fernando concedidas a nuestra Aviación Militar desde su creación en 1913 hasta nuestros días, 11 lo fueron en la Campaña de Marruecos y las otras 6 en la Guerra Civil.

\section{Los grandes vuelos de la Aviación Española}

$\mathrm{Al}$ poco de finalizar la Guerra Europea, en 1919, comenzaron a efectuarse lo que se denominaron Grandes Raids Internacionales. Durante el conflicto, los contendientes habían ido diseñando y fabricando aviones capaces de transportar cargas cada vez de mayor peso y a mayores distancias, lo que permitió al finalizar la contienda poder utilizar la Aviación con unos fines pacíficos que facilitasen la unión entre los pueblos y el comercio mundial, pero al mismo tiempo permitió a los aviadores en- 
frentarse a un viejo desafío: la travesía del Océano Atlántico. Sin embargo, España estaba inmersa en la Campaña de Marruecos, que exigía que todo el esfuerzo aéreo de la nación tenía que dedicarse al apoyo de las operaciones militares a fin de poder pacificar el territorio.

Por ello, cuando en el otoño de 1925 finalizaron con éxito las operaciones en Alhucemas, los aviadores españoles elevaron al jefe de la Aeronáutica Militar un proyecto en el cual se presentaba la realización de tres grandes raids a otras tantas regiones de la Tierra muy entrañablemente unidas a la Historia y al presente de España: Argentina, Filipinas y la entonces colonia española de Guinea Ecuatorial.

El autor del proyecto que tenía destino Buenos Aires fue el comandante Ramón Franco Bahamonde, que estaba considerado como uno de los más grandes aviadores españoles y eligió como aeronave para el vuelo un hidroavión Dornier Wal, que recibiría el nombre de «Plus Ultra». Formaron parte de la tripulación el capitán de Artillería, Julio Ruiz de Alda y como mecánico el soldado Pablo Rada Ustarroz, uniéndose a ellos el teniente de navío aviador Juan Manuel Durán. El vuelo se inició en Palos de la Frontera el 22 de enero de 1926 y finalizaría en Buenos Aires el 10 de febrero siguiente después de recorrer 10.135 kilómetros en ocho etapas y recalar entre otros puntos en Canarias, Río de Janeiro y Montevideo. Fue la primera travesía del Atlántico Sur con un solo avión.

El segundo de estos grandes raids ha pasado a la Historia como Vuelo Madrid-Manila y fue realizado por la «Patrulla Elcano», inicialmente compuesta por tres Breguet XIX, pilotados por los capitanes Martínez Esteve, Loriga Taboada y González Gallarza, acompañados por sus respectivos mecánicos, Calvo Alonso, Pérez Sánchez y Arozamena Postigo, siendo el trayecto previsto de unos $17.100^{\prime \prime}$ kilómetros que esperaban cubrir en 18 etapas. Salieron de Cuatro Vientos el 5 de abril pero desgraciadamente en el trayecto más largo, entre El Cairo y Bagdad, de 900 kilómetros, Esteve y Calvo se vieron obligados a efectuar un aterrizaje forzoso en el desierto sirio, donde estuvieron perdidos seis días, por lo que no pudieron continuar el raid. Igualmente Loriga y Pérez tuvieron que aterrizar en la localidad china de Tien Pack por parada de su motor, mientras González Gallarza llegaba en solitario a Macao, y se convertiría en el héroe del histórico vuelo. Gallarza acompañado en su avión por Loriga aterrizó en Manila el 13 de mayo de 1926 en medio del entusiasmo popular del pueblo filipino.

El vuelo que tenía como meta Guinea Ecuatorial fue efectuado por tres Dornier Wal y la escuadrilla fue denominada «Patrulla Atlántida». Las tripulaciones las componían 12 aviadores, siendo los pilotos el comandante Llorente Solá y los capitanes Martínez Merino y Rubio García. 


\section{La Aviación Militar Española}

La finalidad del vuelo, además de unir por aire a España con su colonia era de carácter científico con el propósito de realizar un levantamiento fotográfico y cartográfico del territorio. La patrulla salió de Melilla el $10 \mathrm{de}$ diciembre y llegó a Santa Isabel el día de Navidad, emprendiendo el vuelo de regreso el 26 de enero de 1927 y amerizó en Melilla el 26 de febrero después de haber recorrido un total de 15.000 kilómetros.

En el año 1928 dos aviadores españoles, los capitanes Iglesias Brage y Jiménez Martín intentaron batir el récord mundial de distancia con un avión Breguet XIX Gran Raid fabricado por Construcciones Aeronáuticas. A pesar de fracasar en su intento al cubrir solamente 5.100 kilómetros, ambos realizarían un nuevo vuelo de 22.000 kilómetros que comprendería Brasil, Uruguay, Argentina, Chile, Perú, Panamá, Nicaragua, Guatemala y Cuba y que sería efectuado en 12 etapas. El avión bautizado como «Jesús del Gran Poder», salió de Sevilla el 24 de marzo de 1929 y tras recorrer la distancia antes citada aterrizaron en La Habana el 17 de mayo. Hay que destacar que en el trayecto entre Santiago de Chile y Lima fueron portadores del documento jurídico que zanjaba definitivamente la cuestión de Tacna y Arica que enfrentaba a Chile y Perú. En 1933 Iglesias se convirtió en el primer jefe militar de una Misión de Paz Internacional, auspiciada por la entonces Sociedad de Naciones a fin de resolver la cuestión de Leticia que disputaban Perú y Colombia.

Con el advenimiento de la II República la Aviación Militar española reanudó los Grandes Vuelos, destacando los realizados a Guinea y Cuba. El primero de ellos lo protagonizaron el capitán Cipriano Rodríguez y el teniente Carlos de Haya en 1931 al cubrir en vuelo directo la distancia Sevilla-Bata en 27 horas y 10 minutos. Habían despegado de la ciudad andaluza el 24 de diciembre a las 10 de la mañana y aterrizaron en la capital guineana a las 2 de la tarde del día de Navidad. Ambos aviadores habían batido el año anterior los récords del mundo de velocidad sobre 5.000 y 2.000 kilómetros, utilizando para ello un Breguet XIX Gran Raid.

El otro vuelo histórico lo realizarían el capitán Mariano Barberán y el teniente Joaquín Collar. Barberán era el mejor navegante y observador aéreo de la Aviación Militar y había planeado con Ramón Franco en 1926 el vuelo del «Plus Ultra», proyectado ese mismo año un vuelo directo Sevilla-Bata que no llegó a materializarse y estudiado el batir los récords de velocidad antes señalados. En esta ocasión se pretendía el vuelo directo entre Sevilla y Cuba para así cruzar con avión por vez primera en la Historia el Atlántico Central. Para este increíble raid utilizaron un Breguet XIX tipo Super Bidón, que construyó Construcciones Aeronáuticas, que fue bautizado como «Cuatro Vientos». Los dos aviadores despegaron de Sevilla el 10 de junio de 1933 y tras cubrir 7.895 kilómetros y volar ininte- 
rrumpidamente durante 39 horas y 55 minutos aterrizaron en Camagüey. $\mathrm{Al}$ día siguiente volaron a La Habana donde permanecieron hasta las primeras horas del 11 de junio, fecha en la que emprendieron el vuelo a México capital, desapareciendo en el trayecto sin dejar rastro. La incógnita sobre como y donde ocurrió la tragedia continúa hasta el día de hoy.

No se debe dejar en el olvido otras grandes gestas de nuestros aviadores, en esta ocasión protagonizadas por pilotos civiles y además en solitario. Las primeras en el tiempo las protagonizó el joven de 30 años Fernando Rein Loring en 1932 y la repetiría en 1933, al volar el mismo trayecto desde Madrid a Manila. En la primera ocasión lo efectuó con una avioneta Loring E-II a la que le puso el nombre de La Pepa. En la segunda realizó la hazaña con una Comper Swift bautizada Ciudad Manila y cubrió la distancia en tan solo 12 etapas e invertir 82 horas. En 1935 se llevó a cabo el último de nuestros Grandes Vuelos, siendo el piloto un joven santanderino de tan solo 21 años, Juan Ignacio Pombo Alonso-Pesquera. Con una pequeña avioneta Eagle, con el nombre Santander, efectuó un vuelo increíble, saliendo de la capital montañesa el 13 de mayo y cruzó el Atlántico Sur en seis etapas, llegando a la costa brasileña el 21 de mayo. Después continuó el raid a la Guayana holandesa, Venezuela, Colombia, Panamá Costa Rica, El Salvador, Guatemala y México, donde llegaría a su capital el 16 de septiembre, tras sufrir diversas incidencias, superar serias dificultades e incluso tener que ser operado de apendicitis en San José, la capital costarricense.

\section{La Aviación Española en la Guerra Civil}

Durante la II República la Aviación española había experimentado un notable desarrollo y no solo en el aspecto técnico. En el año 1932 fue creada la Revista de Aeronáutica, que aglutinaba todas las publicaciones de los organismos aeronáuticos de la Administración y que vino a reemplazar a Aérea, la primera revista española de aviación fundada en 1923 por Kindelán, Emilio Herrera, La Cierva y Torres Quevedo entre otras personalidades. Durante los años previos a la Guerra Civil hubo una gran controversia sobre la necesidad de crear un tercer Ejército independiente del Ejército de Tierra y de la Marina, postura defendida por notables aviadores y pensadores aeronáuticos como Manzaneque, Pastor Velasco, Martínez Merino, Spencer y Fernández Longoria que era a la sazón el director de Revista Aeronáutica, frente a los militares de Tierra y marinos que pretendían continuase la Aviación subordinada a sus respectivos Ejércitos. En este ambiente doctrinal se llegó al 18 de julio de 1936. 
La contienda daría paso a solucionar de una manera positiva las aspiraciones de los aviadores españoles, al menos desde el punto de vista orgánico, pues el Gobierno republicano creó un Ministerio de Aire y Marina y ponía a todas las Fuerzas Aéreas bajo un solo mando en la persona de Hidalgo de Cisneros. Por el lado nacional se creó una Jefatura de los Servicios del Aire, cargo para el que fue designado el entonces general Alfredo Kindelán. Hay seis aspectos importantes a destacar en nuestra Guerra Civil desde el punto de vista aeronáutico: El Puente Aéreo del Estrecho, el Apoyo a las fuerzas terrestres, la lucha por la Superioridad Aérea, el Abastecimiento Aéreo, el Bombardeo y la Acción sobre el mar.

El Puente Aéreo del Estrecho fue la primera operación de este género en toda la Historia mundial de la Aviación y fue una gran sorpresa estratégica y táctica de los nacionales. Como consecuencia de una reunión de altos mandos convocada en Tetuán, a la que asistió Kindelán, Franco adoptó la decisión de transportar por aire a Sevilla a las fuerzas estacionadas en Marruecos con los escasos medios aéreos disponibles. A finales de julio y con la llegada de 20 aviones JU-52 recibidos de Alemania y unos cuantos Savoia-81 enviados por Mussolini, el total de soldados con sus equipos transportados fue de 2.063. A principios de noviembre se dio prácticamente por concluida la operación iniciada el 19 de julio, en la que se habían trasladado un total de 23.393 hombres. La operación pudo y debió haber fracasado pues la aviación republicana disponía en esos meses de la superioridad aérea, pero esta decidió concentrar sus medios aéreos en la sierra de Guadarrama.

La falta de artillería pesada y de acompañamiento, así como de unidades blindadas, hicieron obligado durante toda la guerra el empleo de la Aviación para facilitar el avance de las fuerzas terrestres. La Aviación nacional perfeccionó una táctica ya experimentada en Marruecos contra los rifeños a la que se bautizó como la cadena, que además de afectar a la moral del enemigo y aumentar la propia, proporcionaba unos resultados notables. Por su parte los republicanos tuvieron una destacada actuación en la conocida Batalla de Guadalajara en la que ocasionaron el desastre de los italianos del general Roatta.

La Superioridad Aérea la ejercerían inicialmente los pilotos republicanos, pero la recepción por los nacionales de He-51 alemanes y Fiat CR32 italianos, inclinó la superioridad a su favor, si bien de forma temporal pues en noviembre de 1936 la URSS envió un importante cuerpo aéreo expedicionario en ayuda de la República, en el que figuraban los velocísimos bombarderos bimotores Katiuskas, los cazas biplanos I-15 o Chatos y los nuevos monoplanos I-16 llamados Moscas o Ratas, estos últimos muy superiores a los CR-32 italianos. 
A lo largo de la guerra hubo enfrentamientos aéreos muy notables entre ambos bandos, debiendo destacarse la llamada Batalla Aérea del Jarama, en la que tres aviadores nacionales, García Morato, Salvador y Bermúdez de Castro, se enfrentaron el 18 de febrero de 1937 a 36 cazas republicanos. El ejemplo de los tres pilotos españoles arrastró a los indecisos italianos lo cual permitiría que la victoria se inclinase a favor de los nacionalistas. No ha sido posible cuantificar el número de derribos que hubo durante toda la contienda. Desde el lado nacional, los españoles se atribuyeron 294 victorias aéreas, la Legión Cóndor alemana 314, mientras que la Aviación Legionaria elevaría a 703 los derribos obtenidos, lo cual parece exagerado. Por parte republicana las victorias aéreas fueron igualmente numerosas pero algo inferior a las de los nacionalistas.

El Abastecimiento Aéreo fue efectuado en su mayor parte por los nacionales, sobresaliendo dos actuaciones memorables, la efectuada en ayuda del Alcázar de Toledo y sobre todo la ejecutada en socorro del Santuario de Santa María de la Cabeza. En este último caso se lanzaron desde el aire 100 toneladas de víveres, medicamentos y munición en 166 vuelos, aunque no pudo evitarse que cayera en manos republicanas. El héroe de estos vuelos fue el capitán Carlos de Haya que realizaría 86 del total de las misiones de aprovisionamiento.

La mayoría de las capitales de provincia recibió algún ataque aéreo y aunque Madrid, Oviedo y Teruel sufrirían más continuadamente los bombardeos, sería Barcelona la que tuvo mayor número de víctimas. Sin embargo, desde el punto de vista propagandístico sería el bombardeo de Guernica el que alcanzó mayor resonancia dentro y fuera de España. Guernica fue atacada el 26 de abril de 1937 por 40 aviones de la Legión Cóndor que lanzaron unas 25 toneladas de bombas que destruyeron el 20 por ciento de la localidad, si bien el número de víctimas mortales sería muy bajo pues se elevó solamente a 126, cifra muy lejana de la que circuló en el extranjero. Se estima que a lo largo de la contienda los bombardeos por ambos bandos no alcanzaron la cifra de 10.000 muertos y conviene recordar que desde el punto de vista nacional se pretendió siempre «limitar los daños y destrucciones a las estrictamente indispensables, con el fin de facilitar la reconstrucción nacional al término de la contienda».

La acción sobre el mar fue desarrollada principalmente por la Aviación Nacional que pretendía reducir los abastecimientos de la República atacando a su flota, instalaciones marítimas y buques mercantes. En este sentido las unidades aéreas estacionadas en las Islas Baleares tuvieron la actuación más destacada y en una de esas acciones moriría el héroe del «Plus Ultra», Ramón Franco Bahamonde. 
La Guerra Civil española fue escenario de nuevas doctrinas aéreas, y ensayos de nuevas tácticas y de material aéreo que serían aplicadas en la II Guerra Mundial, obteniéndose lecciones provechosas de la actuación de los dos bandos aunque italianos y alemanes sacarían algunas conclusiones erróneas.

\section{Creación del Ejército del Aire}

Durante la Guerra Civil la Aviación había alcanzado un elevado prestigio y la victoria de los nacionales se debió en gran medida a la actuación de las fuerzas aéreas, siendo opinión generalizada que sería la Superioridad Aérea la que había decidido el resultado final de la contienda. Por esta razón, los aviadores esperaban que había llegado el momento de que la Aviación fuese elevada al mismo nivel que el Ejército y la M rina. Este deseo se vería cumplido en el verano de 1939, cuando una Ley de la Jefatura del Estado fechada el 8 de agosto modificaba la Administración Central del Estado y por la cual desaparecía el Ministerio de Defensa que había sido creado en enero de 1938 y era sustituido por los Ministerios del Ejército, de Marina y del Aire. Sin embargo, la designación del Ministro causó una gran sorpresa y en cierto modo decepción, pues todos esperaban que hubiera sido Alfredo Kindelán, a la sazón ya general de división, sin embargo el cargo lo ocupó un general de brigada no aviador, aunque eso sí, gozaba de cierto prestigio, fue Juan Yagüe Blanco.

Yagüe demostró una gran capacidad organizativa y una enorme actividad legislativa, para lo cual contó con uno de los más prestigiosos aviadores y probablemente el más completo de toda la Aviación española, el entonces teniente coronel Eduardo González Gallarza, el protagonista principal del vuelo Madrid-Manila. Poco después, otra Ley de la Jefatura del Estado de fecha 7 de octubre daba nacimiento al Ejército del Aire (integrado lógicamente en el nuevo Ministerio) que en su primer artículo disponía: «.... Compuesto de mandos, tropa, elementos y servicios regidos por leyes y disposiciones especiales y comunes a los Ejércitos de Tierra y Mar, con los que ha de cooperar a la defensa e integridad de la Patria, al logro de los ideales nacionales y a mantener el imperio de las leyes».

En los siguientes artículos se establecía que en tiempo de guerra el mando correspondía al Generalísimo Franco quien lo delegaba en el Ministro en tiempo de paz y su composición incluía el Estado Mayor General, las Armas de Aviación y Tropas de Aviación, Cuerpo de Ingenieros Aeronáuticos y los Servicios de Ingenieros, Intendencia, Sanidad, Jurídi- 
co, Eclesiástico e Intervención. Complementarias de esta ley serían otras disposiciones inmediatas que la desarrollaban y en las que el Arma de Aviación era considerada como «fundamento, médula y razón de ser» del Ejército del Aire, y recibía la misión de «desarrollar todo su poder ofensivo mediante sus unidades aéreas». Estas se organizaban en escuadrillas, grupos, regimientos y eventualmente brigadas, divisiones, cuerpos de ejército y ejército aéreo. Como puede observarse el legislador conservaba un claro mimetismo con el Ejército de Tierra, siendo igualmente idénticas las divisas y empleos, aspectos estos que no se correspondían con los tradicionales que habían existido anteriormente en nuestra Aviación Militar.

Una de las primeras preocupaciones de los responsables del nuevo Ministerio del Aire fue mantener en vuelo la numerosa y variada flota de aviones existente al terminar la Guerra Civil, que procedentes de la Aviación Nacional disponía de un número aproximado de 350, más 164 capturados o recuperados de la Aviación República, 148 de la Aviación Legionaria italiana y otros 76 de la Legión Cóndor, de los cuales 450 eran aviones de combate y que desfilaron en la histórica Parada de Barajas el 12 de mayo de 1939.

El plan de Yagüe tenía como objetivo alcanzar el número de 5.000 aviones, pero se encontraba inicialmente con nada menos que 96 modelos diferentes, razón por la cual se pensó reducirlos a menos de una docena con la sustitución de los más anticuados por otros fabricados bajo licencia y la importación de Alemania e Italia, pero el comienzo de la II Guerra Mundial frustraría la realización del ambicioso plan. En el campo de la enseñanza Yagüe realizó una gran labor con la creación de diversos centros y escuelas a fin de obtener el personal necesario, pues en 1939 apenas alcanzaba el millar de pilotos.

Curiosamente, cuando el 27 de junio de 1940 llegaban las tropas alemanas a la frontera hispano-francesa fue cesado el general Yagüe y relevado por el entonces general jefe del Alto Estado Mayor Juan Vigón Suerodíaz que mantuvo como jefe del Estado Mayor al ya coronel Eduardo González Gallarza. La tarea de Vigón fue ardua y dura pues la situación internacional creada impedía la renovación del material a lo que se unía la escasez de combustible, lo cual a su vez dificultaba tanto la formación como el entrenamiento de las tripulaciones. La organización territorial recuperó las regiones y zonas aéreas al disponerse del suficiente número de generales y coroneles para el mando de las mismas, a los que se les dió atribuciones análogas a las de los generales jefes de Región Militar o almirantes jefes de Departamento. Las Regiones Aéreas eran la Primera (Centro), Segunda (Estrecho), Tercera (Levante), Cuarta (Pirenaica) y Quinta (Cantábrico) y tres Zonas, Baleares, Canarias y Marruecos. 
En lo relativo a la industria aeronáutica el 7 de mayo de 1942 se constituyó el Instituto Nacional de Técnica Aeronáutica con sede en Torrejón de Ardoz y un año más tarde se adjudicaron a Construcciones Aeronáuticas y a la Hispano Aviación los concursos para fabricar aviones de bombardeo y de combate. En cuanto al personal en 1945 se finalizarían las obras de la nueva Academia General del Aire, en una base aérea construida junto al Mar Menor a comienzos de los años 30, llamada San Javier.

\section{La Escuadrilla Azul}

El Gobierno español decidió ser «no beligerante» en los comienzos de la II Guerra Mundial, a pesar de las intensas presiones de las potencias del Eje, principalmente Alemania, sobre todo a partir de 1940, momento en los que dominaban toda la Europa continental occidental con excepción de la Península Ibérica. En este contexto, en octubre de 1940 se celebró en Hendaya la histórica entrevista del Jefe del Estado español general Franco con el canciller alemán Adolf Hitler. Allí el estadista militar español expuso una serie de condiciones y exigencias para nuestra entrada en la guerra que desalentarían a los dirigentes del III Reich a que sus fuerzas entraran en España para ocupar Gibraltar. Pero cuando en 1941 se produjo la invasión alemana de la URSS, España decidió el envío de una división de infantería de voluntarios y una escuadrilla aérea que recibiría el sobrenombre de Azul, toda vez que la Unión Soviética había tenido una activa participación en nuestra Guerra Civil con una masiva ayuda de hombres y material a la zona republicana.

La unidad aérea estaba compuesta por 20 pilotos de plantilla que eran relevados cada seis meses, apoyados por diverso personal auxiliar y un centenar de hombres entre suboficiales y tropa. La Escuadrilla Azul entró en combate el 2 de octubre de 1941 integrada en el VIII Cuerpo Aéreo de Von Richtofen que había sido el último jefe de la Legión Cóndor en nuestra Guerra Civil. La Unidad tuvo un total de 88 pilotos durante su actuación en el frente oriental hasta que fue retirada por orden del Gobierno en marzo de 1944, perdiendo en combate 19 hombres más 1 prisionero, lo que representa el 23 por ciento del total. Los pilotos españoles tuvieron una decidida y heroica actuación y con sus Messersmicht 109 derribaron al menos 161 aviones soviéticos comprobados, obteniendo trece de ellos la categoría de as, al haber alcanzado cinco o más victorias aéreas.

Por otra parte, debe citarse la presencia de otro grupo de aviadores españoles que combatieron igualmente en el frente soviético. En este 
caso fueron entre 80 a 90 pilotos procedentes de la anterior Fuerza Aérea de la República que participarōn en defensa de la URSS, la mayoría en unidades de caza. Aproximadamente un 30 por ciento de los aviadores españoles republicanos murieron en combate, estimándose que unos 20 podrían haber obtenido la categoría de as.

Esta sería la primera intervención aérea en una guerra de aviadores españoles después de nuestra contienda civil.

\section{El difícil quinquenio 1945-1950}

Al terminar la II Guerra Mundial, en el verano de 1945, comenzarían unos años muy difíciles para España, ya que junto a la difícil tarea de reconstruir el país después de la Guerra Civil y de la escasez habida durante el grave conflicto internacional, hubo que añadir el aislacionismo y bloqueo económico a los que fue sometida nuestra Patria por parte de las recién creadas Naciones Unidas de las que fuimos excluidos. En esa situación el nuevo general de división Eduardo González Gallarza Iragorri era nombrado Ministro del Aire en sustitución de Vigón. Era el primer aviador que llegaba a ocupar el más alto cargo en la Aviación española y ya desde el primer día demostró una gran intuición pues designó como Jefe del Estado Mayor del Aire a un prestigioso aviador y uno de los mejores pensadores aeronáuticos que ha habido en España, Francisco Fernández y González Longoria. Ambos formarían un excelente equipo para enfrentarse a los graves problemas existentes y a otros en ciernes, pues tenían que resolver la grave situación originada por el envejecimiento del material, a crear una infraestructura aérea adecuada y a modernizar y adecuar la organización del Ejército del Aire a las nuevas corrientes de la doctrina aérea.

Por otra parte, la falta de personal se había venido solucionando lo que facilitó que a partir de 1946 se creasen los Servicios de Automovilismo y de Transmisiones, se constituyera la Primera Bandera Paracaidista; al año siguiente comenzaba a funcionar la Milicia Aérea Universitaria y la Escuela Militar de Paracaidismo. En 1948 se fundaron la Escuela de Cartografía y Fotografía y el Servicio de Armamento y en 1950 la Escuela de Transmisiones.

El excesivo número de tipos de aviones existentes había obligado a que básicamente se fuera orientando a conservar los Ju-52 como avión de transporte, los He-111 como plataformas de bombardeo y de reconocimiento, los Me-109 serían la base de la caza y las avionetas Bücker instrumento de enseñanza básica, aunque todavía quedarían algunos cazas 
Fiat CR-32 e I-15 y los H5-42 y 43, entre otros. Aunque la industria aeronáutica española no había tenido problemas muy graves para fabricar las células de los aviones, sí los hubo en cambio para encontrar las plantas motrices adecuadas, en particular para los He-111 y Me-109, hasta que la solución llegó con la adquisición en 1951 de motores Rolls Royce Merlin.

Pero González Gallarza prestaría una gran atención al desarrollo de la Aviación Civil, potenciando el transporte aéreo y la red aeroportuaria, cuyo despegue se iniciaría al asistir una delegación española a la inauguración de la Asamblea General de la Organización Internacional de Aviación Civil (OACI) que tuvo lugar en Chicago en noviembre de 1944. La compañía de bandera española Iberia había nacido al amparo de la Ley de 7 de junio de 1940 que le concedía el monopolio del transporte aéreo regular de pasajeros, correspondencia y mercancías cuyas primeras aeronaves fueron básicamente los Ju-52 y algunos DC-2, pero al recibir en 1944 los primeros Douglas DC-3 iniciaría una tímida expansión. Su crecimiento no comenzaría hasta dos años después, cuando en 1946 adquirió los primeros Douglas DC-4 que le permitiría establecer una línea con Buenos Aires y en 1948 su flota llegaba ya a los 25 aviones. Ese año se creaba una segunda compañía aérea, Aviación y Comercio y en 1952 el tráfico aéreo de pasajeros se había multiplicado por diez en relación a 1940, el de mercancía por seis y el de correo por dos. Estos índices crecerían mucho más espectacularmente con la potenciación de la red aeroportuaria.

Efectivamente, a partir de 1945 la Dirección General de Aeropuertos reformó notablemente las instalaciones de los cuatro aeropuertos más importantes, Madrid, Barcelona, Sevilla y Valencia e inició la construcción de una amplia red que en 1953 se elevaba a 26 aeropuertos, más otras nueve bases y aeródromos militares abiertos al tráfico civil, lo que permitía operar además de las compañías españolas a 18 líneas aéreas extranjeras. Gallarza y Longoria tampoco olvidaron la aviación deportiva y el vuelo a vela pues ambos sabían perfectamente que son dos viveros de vocaciones aeronáuticas. Los éxitos de Luis Juez, con su récord del mundo de altura en 1947 al subir con un velero a 6.263 metros y los de Miguel Ara, hicieron posible que España fuera por vez primera sede de unos campeonatos del mundo de vuelo sin motor que tuvieron lugar en 1952 y en los cuales Juez volvería a obtener un título mundial.

\section{La modernización de la Aviación Española}

Al derogar la Asamblea General de las Naciones Unidas el 4 de noviembre de 1950 su resolución por la que había recomendado la retirada 
de embajadores de Madrid, ese mismo mes Estados Unidos solicitaba el restablecimiento de relaciones diplomáticas con España y el nombramiento de embajadores y con la designación de representantes diplomáticos de otras naciones se daría fin a la penuria de material y de combustible que venía impidiendo el desarrollo de la Aviación.

En mayo de 1951 el Ministro del Aire, González Gallarza, se trasladó a Manila invitado por el gobierno filipino para conmemorar el 25 aniversario de su histórico vuelo a las islas efectuado en 1926 y con tal motivo el Secretario de Estado norteamericano le invitó a que visitase diversas bases e instalaciones militares y aéreas y los más importantes centros industriales aeronáuticos del país. La llegada de un prestigioso general a la Casa Blanca con la designación de presidente de los Estados Unidos de Eisenhower y el estallido de la Guerra de Corea habían hecho recapacitar a la gran nación americana sobre la necesidad de incorporar a España al sistema defensivo de Occidente. Sin embargo, la resistencia de algunos países miembros de la recién creada OTAN a que España ingresara en la Organización llevó a que Eisenhower ofreciese iniciar conversaciones con el gobierno del Generalísimo Franco con el objeto de firmar unos tratados de cooperación.

Las enseñanzas de la II Guerra Mundial y el vertiginoso desarrollo de la industria aeronáutica aconsejaban modernizar la organización del Ejército del Aire y adaptarla a la nueva situación. Con tal motivo el Ministro propuso al Gobierno la remisión de una ley a las Cortes que sería aprobada el 15 de julio de 1952, por la cual las Fuerzas Aéreas se estructuraban en:

- Aviación de Defensa Aérea, con la misión de proteger el espacio aéreo y territorio nacional contra ataques enemigos.

- Aviación Táctica, responsable de cooperar y apoyar al Ejército de Tierra y la Marina.

- Aviación Estratégica, encargada de la acción aérea ofensiva.

- Aviación de Transporte para efectuar los del personal, material y abastecimientos.

Se fusionaban las Armas de Aviación y las de Tropas de Aviación y los Servicios que se establecían eran de Transmisiones, Cartografía y Fotografía, Armamento, Combustible, Automóviles, Defensa Química y Contraincendios, así como otros que pudieran crearse en el futuro. Las unidades aéreas, incluidas las de Tropas y Servicios, pasaron a denominarse Patrulla, Sección, Escuadrilla, Escuadrón, Grupo, Ala, División y Fuerza Aérea.

El 24 de septiembre de 1953 se firmaron entre España y Estados Unidos tres Convenios íntimamente relacionados: 
- Convenio de Ayuda para la Mutua Defensa, con vigencia indefinida y que facilitaba ayuda militar a España.

- Convenio de Ayuda Económica que regulaba la ayuda a España para la adquisición de bienes y servicios en Estados Unidos.

- Convenio Defensivo, por lo cual Estados Unidos se comprometían a facilitar material y equipos militares para modernizar las Fuerzas Armadas españolas y establecer un moderno sistema de Defensa Aérea. Por su parte España cedía el uso de las bases aéreas de Morón, Torrejón y Zaragoza, la aeronaval de Rota y una amplia red de estaciones de Comunicaciones, todas ellas a construir por los Estados Unidos, pero bajo jurisdicción española.

A comienzos de 1954 el Ejército del Aire disponía de unos 900 aviones de los cuales solamente estaban en servicio unos 600, de los que 30, aunque estaban catalogados como cazas realmente no podían operar como tales. El número de aviones de enseñanza superaba los 350 y el resto eran de transporte, bombardeo, reconocimiento y enlace. Esta era la flota que había que renovar. Un gran número de aviones comenzaría a causar baja con la llegada del material aéreo norteamericano, pero todavía durante cierto tiempo tuvieron que coexistir dos flotas, la nueva y otra antigua que aunque en principio serviría para la enseñanza. Sin embargo, tendría que actuar, como se verá más adelante, en acciones de guerra.

A fin de preparar al personal de vuelo que debería tripular y mantener el nuevo material, comenzó a enviarse a bases americanas en Alemania y Estados Unidos un gran número de oficiales y suboficiales a partir de abril de 1954. Pocos días antes llegaron los primeros reactores de enseñanza y entrenamiento T-33 a la base aérea de Talavera Real, que aún estaba en obras y durante el verano de ese año llegaron a la base aérea salmantina de Matacán, los de hélice T-6D de enseñanza básica.

Por la misma época se recibieron los Grumman de salvamento y en junio de 1955 aterrizaban en Getafe los primeros reactores de combate F-86F.

La llegada del nuevo material hizo obligada la creación de nuevas unidades y la desaparición de otras. Así nacería en septiembre de 1955 el Ala de Caza número 1 que quedaba estacionada en la base aérea de Manises, en Valencia, un año más tarde se constituyó la número 2 con base en Zaragoza y en febrero de 1957 se creaba la número 3 con sede en Valladolid. A ellas seguirían otras unidades aéreas de caza, transporte, reconocimiento y enlace así como el escuadrón de Alerta y Control.

El 13 de abril de 1956 se creaba el Mando de la Defensa Aérea, de carácter ínter ejércitos cuyo primer jefe fue el teniente general del Ejército del Aire Julián Rubio López. Hay que resaltar igualmente que ese mismo año se constituía el Servicio de Búsqueda y Salvamento, más co- 


\section{José Sánchez Méndez}

nocido por sus siglas SAR con la misión de localizar las aeronaves siniestradas y prestar los primeros auxilios a sus tripulantes y pasajeros.

La llegada del material norteamericano serviría también para impulsar nuestra industria aeronáutica que venía modernizando el material convencional que aún se conservaba y que comenzaba a diseñar sus propios aviones como fueron entre otros el de transporte C-207 Azor de Construcciones Aeronáuticas y el HA-200 Saeta de la Hispano Aviación, que en este último caso contaba con el famoso ingeniero alemán profesor Messerchmitt. La Aviación Civil impulsó su expansión con la llegada de aviones con mayores capacidad y autonomía, como los Convair y los Super Constellation que permitieron entre otras nuevas líneas la de Madrid con Nueva York.

Se daba así fin a la etapa más fructífera de la historia de nuestra Aviación, que comprendió los doce años que dirigió el Ministerio del Aire el general Eduardo González Gallarza, el cual sería relevado por otro insigne general, José Rodríguez y Díaz de Lecea, quien a los pocos meses de su toma de posición tuvo que hacer frente al conflicto militar de Ifni que amenazaba extenderse al Sahara español.

\section{La Guerra de Ifni}

El 7 de abril de 1956 España reconocía la independencia del reino de Marruecos en una declaración conjunta hispano-marroquí y se comprometía a retirarse de la zona norte donde había ejercido el protectorado. Pero en el otoño de ese año bandas armadas procedentes de Marruecos se infiltraron a través del Sahara español y en enero de 1957 atacaron un puesto francés en Mauritania. La aparente pasividad española envalentonó a las bandas marroquíes de liberación que el 15 de marzo se reunieron cerca del enclave español de Ifni y acordaron expulsar a los extranjeros en nombre del Sultán.

España desoyó una propuesta francesa de actuar conjuntamente y decidió expulsar a las bandas que llegaban ya a los tres mil hombres. El fuego de estos contra aviones militares españoles de reconocimiento obligó a reforzar desde la Península a las unidades aéreas de Canarias, que era desde donde se efectuaban los vuelos, pero el incremento de las agresiones se contestó con bombardeos aéreos con B.2I (He-111 de fabricación nacional). Para reforzar nuestras posiciones en Ifni y en los más importantes núcleos del Sahara se realizó una importante operación de transporte aéreo con DC-3 y JU-52 que trasladaron tropas de refuerzo y varias toneladas de material a El Aiun y Sidi Ifni. 
El 23 de noviembre los marroquíes lanzaron un importante ataque contra Ifni por lo que se aerotransportó desde El Aiun a la VI Bandera de la Legión. Ante la falta de munición de las armas de a bordo de los $\mathrm{He}$ 111 se solicitó el apoyo de Francia que desoyó la petición, sin embargo los JU-52 intensificaron sus bombardeos y los aviones del Ala 35 de transporte abastecieron de agua, munición y víveres a la capital y a otros enclaves españoles sitiados. Es de destacar la llamada Operación Pañuelo destinada a reforzar Tiliuín con tropas paracaidistas que saltaron desde cinco Ju-52. Rotos los cercos de esta posición y la de Telata se dispuso fortalecer las defensas de Ifni. Pero al finalizar estas operaciones, el 30 de noviembre se produjeron en el Sahara dos importantes agresiones marroquíes, en una de las cuales secuestraron al destacamento del Cabo Bojador y a mediados de diciembre sorprendieron a la III Bandera de la Legión a la que le causaron fuertes bajas. Por acuerdo hispano-francés se constituyeron una agrupación de cada país y con lanzamiento de fuerzas paracaidistas se alcanzaron los objetivos de pacificar El Sahara. La Bandera Paracaidista del Ejército del Aire lo haría desde aviones franceses que despegaron de Mauritania.

Nuestros compañeros del Ejército de Tierra calificaron a nuestros aviadores como el elemento fundamental y decisivo para la obtención del éxito, pero la guerra puso de relieve la falta de medios aéreos adecuados, armamento moderno y entrenamiento de las tripulaciones que derrocharon en todo momento un altísimo valor y heroísmo. Las operaciones logísticas recayeron en $12 \mathrm{Ju}-52$, ocho He-111 y 15 DC-3 que realizarían cerca de tres mil horas de vuelo sobre una zona con nulas ayudas a la navegación. A ellos se unirían para las operaciones del Sahara aviones T6 armados con ametralladoras y cohetes que darían un gran resultado y algunos Me-109 fabricados en España que tuvieron poco éxito.

La Guerra en Ifni y Sahara nos mostró un aspecto negativo, probablemente el mayor, de los convenios firmados con los Estados Unidos pues no autorizaron el empleo del nuevo material militar cedido en virtud de dichos acuerdos. En la campaña el Ejército del Aire tuvo 18 muertos y 15 heridos entre tripulantes y paracaidistas.

\section{Nace la red de alerta y control}

Poco después de la firma de los convenios hispano-norteamericanos se comenzó a estudiar los posibles y mejores emplazamientos de los escuadrones de un organismo esencial, pues serían los ojos del Mando de la Defensa Aérea y un instrumento esencial para conducir y apoyara nuestras 
tripulaciones: la Red de Alerta y Control. Entre 1958 y 1960 fueron entrando en servicio los escuadrones que lo componían y que se distribuyeron estratégicamente a fin que sus radares pudieran proporcionar una cobertura que facilitase el despegue de los cazarreactores ante el vuelo de un avión no identificado. Estas unidades fueron situadas en Calatayud, Villatobas, Constantina, Rosas, Alcoy, Elizondo y Soller, desplegados en un sentido NE-SO a fin de poder interceptar más fácilmente eventuales vuelos de los aviones del entonces Pacto de Varsovia. En la Base Aérea de Torrejón se instaló el puesto de mando del jefe de la Defensa Aérea, en el Centro de Operaciones de Combate.

En el mes de septiembre de 1958 se incorporaba a la Academia General del Aire el Príncipe Juan Carlos de Borbón y Borbón, que lo hacía con el empleo de alférez-alumno, pues desde 1955 venía siguiendo su formación militar, primero en la Academia General Militar en Zaragoza y posteriormente en la Escuela Naval de Marín. El 15 de julio de 1959 el Príncipe recibió su título de piloto, habiendo durante su estancia en San Javier formado parte de la escolta de honor de la Bandera. Al finalizar sus estudios pasaría a formar parte de la 11 Promoción de la Academia General del Aire.

En la época de Díaz de Lecea se construyeron pistas asfaltadas en todas las bases aéreas equipadas con reactores y en otras siete muy importantes, modernizándose igualmente sus instalaciones. En 1962 había 33 aeropuertos abiertos al tráfico civil que tuvieron 180.000 vuelos y recibieron cuatro millones y medio de pasajeros, lo que pone de relieve el crecimiento de nuestra Aviación Civil. Junto a Iberia y Aviaco nacieron otras dos compañías aéreas, Tassa y Spantax, llegando esta última a convertirse en una de las empresas de vuelos charter más importantes de Europa.

\section{Renovación Tecnológica. Llegan los Cazas Supersónicos}

Cuando el 10 de julio de 1962 se remodela el Gobierno de la Nación es designado Ministro del Aire el teniente general, José Lacalle Larraga quien permanecería en el cargo siete años y designó como jefe del Estado Mayor al teniente general Martínez Merino que fue el piloto del hidroavión Dornier Wal «Cataluña» de la Patrulla Atlántida. Un año más tarde, en abril de 1963 se realizó la primera demostración de fuego real del Ejército del Aire en el nuevo polígono de tiro de las Bárdenas Reales, en Navarra, en un brillante ejercicio que recibió el nombre de Operación «etrería». 


\section{La Aviación Militar Española}

Siguiendo la política gubernamental de primar el crecimiento económico y social del país, el Ministerio del Aire recibiría el más alto presupuesto de su historia para el cuatrienio 1964-67 con un 33.25 por cien del total de los gastos militares, pero la mitad de dicho presupuesto iría destinado al desarrollo aeroportuario para beneficiar la expansión de nuestro turismo, por lo cual fue creada una Subsecretaría específica para la Aviación Civil. En la historia del Ejército del Aire siempre que hubo cambio en su Jefatura se introdujeron modificaciones en la Organización y en este caso igualmente las hubo, razón por la cual las Regiones Aéreas se redujeron a tres, con cabeceras en Madrid, Sevilla y Zaragoza y una Zona Aérea en Canarias.

A su vez la Aviación Táctica adquiría el rango de Mando Aéreo y su cuartel general y jefatura se vinculaban a la Segunda Región Aérea o del Estrecho. La necesidad de centralizar y coordinar la política de adquisiciones, abastecimientos y mantenimiento derivadas de la creciente complejidad de los nuevos sistemas de armas aéreos aconsejaba la creación de un organismo que aglutinase tales necesidades logísticas, razón por la cual en diciembre de 1965 se constituía el Mando de Material. En la Defensa Aérea nacía un nuevo Escuadrón de Alerta y Control que se emplazó en el Pico de las Nieves en Gran Canaria.

Pero las necesidades de la Defensa Nacional exigían una renovación y modernización del material aéreo y en enero de 1965 llegaban a España los primeros cazas supersónicos F-104 que pasarían a constituir el 161 Escuadrón, estacionados en la base aérea de Torrejón de Ardoz. Los aviones permanecerían en servicio en España hasta 1972 y volaron más de 17.000 horas de vuelo sin registrar ningún accidente. Igualmente en enero de 1965 el Gobierno decidió la adquisición de 70 cazas supersónicos F-5 Northrop que serían fabricados en Construcciones Aeronáuticas con el apoyo tecnológico de la empresa norteamericana. A su vez la aviación de transporte recibía una remesa de aviones Caribou y se creaba la Aviación Antisubmarina que comenzó a disponer de aviones HU-16. Pero la modernización se extendió a una nueva actividad no desarrollada hasta entonces, la espacial; en colaboración con la NASA y la Agencia Europea del Espacio se construyeron las estaciones de Maspalomas, Robledo de Chavela, Villafranca del Castillo y una internacional de comunicaciones en Buitrago.

Hubo en este periodo 1962-1969 algunas fechas memorables como las de septiembre de 1964, cuando el capitán Tomás Castaño alcanzó el primer puesto del III Campeonato del Mundo de Vuelo Acrobático que se celebró en Bilbao o el estreno del Himno oficial del Ejército del Aire, con letra de José María Pemán, que se tocó por vez primera el 10 de diciembre de 1967, día de la Virgen de Loreto Patrona de Aviación. Pero también hubo mo- 
mentos poco agradables, tales como la supresión de la Milicia Aérea Universitaria, la desaparición de la Academia de Ingenieros Aeronáuticos, las disoluciones de la prestigiosa Patrulla Acrobática Ascua y del escuadrón de Paracaidistas, a los que habría que añadir los nacimientos incipientes de las unidades de Aviación en el Ejército de Tierra y la Armada y la pérdida de la condición interejércitos del Mando de la Defensa Aérea.

\section{Llega el nuevo material aéreo francés}

El 22 de julio de 1969 Franco notificaba a las Cortes la designación como Sucesor a la Jefatura del Estado del Príncipe Juan Carlos de Borbón y Borbón y en octubre de ese año es remodelado el Gobierno, pasando a ser Ministro del Aire el general Julio Salvador y Díaz Benjumea. $\mathrm{Al}$ año siguiente y como consecuencia de unos convenios de cooperación militar firmados con Francia, aterrizaban en la base aérea de Manises los primeros Mirage III que en número de 30 fueron adquiridos a aquel país, de los cuales seis serían biplazas para entrenamiento.

Mientras tanto se produjo un hecho que supondría una gran transformación de la industria aeronáutica española, pues los estudios y contactos que venían celebrándose entre Construcciones Aeronáuticas y la Hispano Aviación darían paso a la fusión de ambas empresas, la cual tuvo lugar en abril de 1971 y un año más tarde CASA absorbería a la fábrica de motores de aviación ENMASA. De esta forma se abrían unas excelentes perspectivas para la nueva compañía que venía desarrollando una gran actividad con revisión y modernización de miles de aviones militares norteamericanos. Al mismo tiempo presentaba en el Salón Aeronáutico de París de ese año el primer prototipo del transporte ligero C-212, que con el nombre de Aviocar tuvo un gran impacto en el mercado internacional.

La renegociación de los acuerdos hispanonorteamericanos iniciadas en 1970 permitirían que por el programa de ayuda militar en junio de 1971 comenzaran a llegar a la base aérea de Torrejón los primeros cazas F-4C Phantom de un total de 36 que irían llegando sucesivamente y todavía durante unos meses coexistirían en dicha base con los F-104. Casi paralelamente fue aumentando el número de los aviones F-5 que Construcciones Aeronáuticas estaba fabricando bajo licencia, los cuales se quedarían en la base aérea de Morón mientras que los de doble mando pasaban a la Escuela de Reactores, en Talavera la Real.

Este primer quinquenio de los años 70 se caracterizó por la llegada de diverso material aéreo que vino a potenciar la capacidad operativa del Ejército del Aire. Así, se recepcionaron tres aviones cisternas KC-97 con 
lo que se aumentaba el radio de acción de los Phantom, se adquirieron aviones Mystere-20 para el traslado de autoridades y calibración de las ayudas a la navegación aérea e igualmente el Ala 22 de acción sobre el mar recibiría los primeros aviones antisubmarinos P-3 Orión. Por el contrario se dieron de baja a los últimos e históricos He-111 que, con el cariñoso sobrenombre de «Pedros». habían formado parte del Ejército del Aire desde 1939. En la primavera de 1973 la Aviación de Transporte española recibiría un notable impulso, al crearse en la base aérea de Zaragoza el 301 Escuadrón que al finalizar el año recibió los primeros Hércules C-130, a los cuales les seguirían otros similares pero con capacidad de reabastecer en el aire a la flota de combate. Como hecho a recordar, la desactivación del escuadrón de Alerta y Control de Elizondo y la entrada en servicio de una nueva unidad del mismo tipo en Motril.

Otro hecho notable a destacar es el extraordinario desarrollo de nuestra Aviación Civil, que a comienzos de 1970 registró un número de pasajeros transportados de unos doce millones de pasajeros así como la entrada en servicio de nuevos aeropuertos y el incremento de la capacidad transoceánica de Iberia, y el notable potencial de Spantax y Aviaco.

\section{Juan Carlos I, Rey de España}

En enero de 1974 hubo una completa remodelación del Gobierno como consecuencia del asesinato terrorista del presidente del Gobierno, almirante Carrero Blanco, que sería sustituido por Carlos Arias Navarro. En el nuevo Gabinete ocupó el cargo de Ministro del Aire, el teniente general Mariano Cuadra Medina.

Siguiendo la política de sus antecesores de diversificar la adquisición del material aéreo a otras naciones, el 1 de junio de ese año se constituía el Ala de Caza número 14 cuya base sería la de Los Llanos, en Albacete, y el material asignado fue el caza francés Mirage F-1C, cuyas primeras 15 unidades se recepcionaron entre junio de 1975 y noviembre de 1977. También en el año 1974, el Ejército del Aire comenzó a recibir los aviones de transporte C-212 Aviocar cuya versión inicial fue la de reconocimiento fotográfico por lo que pasaron a prestar servicio en el Centro Cartográfico y Fotográfico, para ya en la primavera del año siguiente entregarse el modelo de transporte a las Alas 35 y 46 con base en Getafe y Gando respectivamente. En esta política activa de renovación y adquisiciones, el 28 de junio de 1975 se recepcionaban los helicópteros SA330 Puma, que formarían el núcleo de una Unidad Especial para transporte de personalidades con base en Cuatro Vientos. Por otra parte 
la evolución internacional aconsejaba revisar y actualizar la Doctrina Aérea, razón por la cual se constituyó una Comisión responsable de llevar a cabo los estudios pertinentes.

Pero 1975 marcaría para siempre la Historia de España con la muerte del Jefe del Estado, Generalísimo Franco, quien ya había sufrido en el verano del año anterior un proceso circulatorio grave del que pudo recuperarse, aunque no totalmente, y en octubre de 1975 comenzaría un deterioro notable de su salud que le llevaría a la muerte el 20 de noviembre. Aunque las previsiones sucesorias estaban claras, pues el Príncipe Juan Carlos había asumido interinamente las competencias de la Jefatura del Estado y tras la muerte del general, fue designado Rey de España, con el nombre de Juan Carlos I, el 22 de noviembre; sin embargo, durante los días previos hubo cierto grado de incertidumbre que sería aprovechado por el rey de Marruecos para lanzar la conocida Marcha Verde para ocupar el Sahara español, que desembocaría en los Acuerdos de Madrid que se firmaron el 14 de noviembre entre España, Marruecos y Mauritania y como consecuencia de los mismos España se retiraba del Sahara. La Marcha Verde había estado vigilada por nuestras unidades aéreas y en la evacuación de nuestras tropas, en una operación que se denominó Golondrina, participaron todos las unidades aéreas de transporte del Ejército del Aire.

A la muerte de Franco hubo que formar un nuevo Gobierno, el primero de la Monarquía, en el que Arias Navarro continuaba de Presidente del Ejecutivo, pero hubo un cambio en el Aire al ser designado Ministro el teniente general Carlos Franco Iribarnegaray. En julio de 1976 cesaba Arias Navarro y ocupó el cargo de Presidente del Gobierno Adolfo Suárez González, quien mantuvo a Iribarnegaray como Ministro del Aire.

Las nuevas circunstancias exigían poner al día la Organización del Ejército del Aire, motivada también porque se habían regulado por un real decreto-ley las atribuciones, funciones y responsabilidad del jefe del Estado Mayor del Ejército del Aire y se había institucionalizado la Junta de Jefes de Estado Mayor, cuyo primer presidente sería un aviador, el teniente general Felipe Galarza Sánchez, que poco después sería sustituido por otro general, igualmente aviador, Ignacio Alfaro Arregui.

La nueva Organización del Ejército del Aire tenía ahora un carácter funcional y no territorial como había sido hasta entonces, y definía a la Fuerza Aérea que se subdividía en mandos aéreos específicos, el de Combate o MACOM, el Táctico o MATAC y el de Transporte o MATRA, que serían apoyados por los mandos logísticos de Personal y de Material. 


\section{Creación del Ministerio de Defensa. Los Programas ORGEA y FACA}

Pero en julio de 1977, Adolfo Suárez reorganizaba la Administración del Estado e introducía un cambio fundamental en la dirección y unificación de las Políticas Militar y de Defensa e integraba en un solo Departamento a los tres Ministerios Militares, con lo que nacía así el Ministerio de Defensa, si bien la subsecretaría de Aviación Civil pasaba a integrarse en el nuevo Ministerio de Transportes y Comunicaciones.

$\mathrm{Al}$ establecerse en noviembre de 1977 la estructura orgánica y funcional del Ministerio de Defensa era necesario adaptar la del Ejército del Aire a la de aquél, razón por la cual el entonces jefe del Estado Mayor del Aire, general Ignacio Alfaro Arregui, creó a finales de 1977 una Comisión que estudiase la nueva organización a constituir, así como las misiones y cometidos de cada uno de los organismos y dependencias del Ejército del Aire. Como resultado de dicho trabajo el 3 de mayo de 1978 un real decreto daba vida al proyecto que se conocería como Programa ORGEA (Organización del Ejército del Aire).

Uno de los aspectos más sobresalientes era que definía al Ejército del Aire como «instrumento militar del Poder Aéreo y un factor esencial de disuasión». Asimismo hay que resaltar que la nueva estructura se basaba en las funciones de los órganos y no en su ubicación territorial, definiéndose tres esenciales:

- La función directiva le correspondía al jefe del Estado Mayor del Ejército del Aire, que para ejercerla contaba con el Cuartel General del Aire.

- La función operativa recaía en la Fuerza Aérea

- La función de apoyo radicaba en la Logística Aérea

Para cumplir su misión la Fuerza Aérea se subdividía en cuatro Mandos Aéreos, Combate, Táctico, Transporte y de Canarias y la Logística Aérea en Mando de Personal, de Material y Dirección General de Infraestructura. Al terminar el año 1978 la Fuerza Aérea contaba con las siguientes unidades más importantes:

- Mando Aéreo de Combate, Ala 11 (Mirage III E), Ala 12 (F-4C Phantom), Ala 14 (Mirage F-1 C) y Ala de Alerta y Control.

- Mando Aéreo Táctico, Ala 21 (F-5, RF-5 y Supersaetas) y Ala 22 (P.3 Orión), más el Grupo Control Aerotáctico y la Escuela de Apoyo Aéreo.

- Mando Aéreo de Transporte, Ala 31 (C-130 Hércules), Ala 35 (C212) y Ala 37 (Caribou DHC-4A).

- Mando Aéreo de Canarias, Ala 46 (F-5A, RF-5A y C-212) 
Por su parte, del Mando de Personal dependían todos los centros de enseñanza y los de asistencia sanitaria, del Mando de Material las tres Maestranzas Aéreas existentes y el resto de las Unidades aéreas de la Agrupación del Cuartel General, que integró entre otras al Servicio de Búsqueda y Salvamento y a la escuadrilla de Autoridades y Calibración de Ayudas a la Navegación que venían dependiendo de la Aviación Civil.

A finales de 1978, Ignacio Alfaro Arregui que había sido designado Presidente de la Junta de Jefes de Estado Mayor, había marcado con anterioridad como Objetivo de Fuerza del Ejército del Aire: doce escuadrones de combate, dos de acción sobre el mar, uno de reabastecimiento en vuelo, dos de transporte medio, cuatro de transporte ligero, nueve de instrucción, más otros varios de misiones diversas, lo que comportaba la finalización de una serie de programas en marcha entre los que había dos fundamentales, la selección de un futuro avión de combate y ataque y la modernización del Sistema Semiautomático de Defensa Aérea.

La selección del futuro avión de combate y ataque (FACA) sería uno de los programas más importantes habidos en la historia del Ejército del Aire y después de una estricta y completa evaluación de los sistemas de armas más avanzados de Europa y Estados Unidos, la decisión final sería adquirir 72 aviones F-18 Hornet.

Durante los años finales de la década de los 70 y comienzo de los años 80 fueron numerosos los movimientos y cambios de unidades e igualmente ocurriría con la baja de aviones de diversos tipos que habían cumplido sobradamente sus ciclos de vida como los históricos JU-52 y DC-3, los T-6, los Saeta y Supersaeta y otros varios. Pero por otro lado se incorporaron nuevos modelos que permitirían mejorar la capacidad operativa de la Fuerza Aérea Española, como fueron dos DC-8 para el transporte de personalidades.

Sería un avión de enseñanza avanzado, diseñado y fabricado por Construcciones Aeronáuticas, una de las grandes estrellas incorporadas a nuestra flota, el C-101 Mirlo. Este magnífico monorreactor pasó a equipar inicialmente a la Academia General del Aire, como parte de un pedido de sesenta unidades, siendo los primeros recepcionados los cuatro prototipos fabricados en febrero de 1979, para continuar la entrega de la serie en 1980. En este último año se instalaba en la base aérea granadina de Armilla la Escuela de Helicópteros que recibiría más tarde el nombre de Ala 78.

\section{España ingresa en la OTAN}

A comienzos del año 1982, siendo Ministro de Defensa Alberto Oliart, fue designado nuevo Jefe del Estado Mayor del Aire el general Emilio 


\section{La Aviación Militar Española}

García-Conde Ceñal y meses más tarde España pasaba a formar parte de la Organización del Tratado del Atlántico Norte, cuyo ingreso sería efectivo el 5 de junio con el acto del izado de la insignia nacional en el Cuartel General de la OTAN, en Bruselas, que lo sería por el brigada del Ejército del Aire José Galán García.

Paralelamente continuaba el programa de adquisición de los F-1 que incluía otros 48 aparatos, pero que disponían de cierta capacidad de guerra electrónica. En 1982 se conmemoraría el 50 Aniversario de la fundación de la Revista de Aeronáutica y Astronáutica y como consecuencia del impulso que había recibido la investigación histórica de la Aviación nació el Servicio Histórico y Cultural que aglutinaba al Instituto de Historia y Cultura Aeronáuticas y al Museo Aeronáutico que había abierto sus puertas dos años antes.

A finales de 1983 voló el primer prototipo de un avión de transporte medio que había sido diseñado por Construcciones Aeronáuticas y la firma indonesia Nurtanio, que recibió la designación CN-235 y del cual el Ejército del Aire recibiría 20 unidades para equipar el Ala 35 en Getafe.

Coincidiendo con la modificación en 1984 de la Ley Orgánica de la Defensa Nacional, el Gobierno aprovechó la circunstancia para designar como nuevo Jefe de Estado Mayor del Aire al general José Santos Peralba Giráldez, que era el primer aviador militar procedente de la Academia General del Aire que llegaba a tan alto puesto. Durante su mandato se recibieron los primeros EF-18, procedentes de los Estados Unidos, que llegarían en vuelo directo y tripulados por pilotos españoles desde las factorías de McDonnell Douglas, por lo que tuvieron que ser repostados ocho veces en el aire, aterrizando en la que inicialmente sería su base de destino, en Zaragoza el 11 de julio de 1986 y como material del Ala 15 que había sido constituida a tal fin. En esas fechas el Ejército del Aire se unía al proyecto de un Futuro Avión de Combate Europeo, el EFA, en el que inicialmente participaban además Alemania, Francia, Italia y Reino Unido. Posteriormente Francia se retiró del Programa que pasaría más tarde a ser denominado Eurocaza o EF-2000. A finales del 1986 el general Peralba pasó a ocupar la jefatura del Cuarto Militar de S.M. El Rey, siendo designado para sustituirle el general Federico Michavila Pallarés, compañero de promoción, ocupando la jefatura del Estado Mayor de la Defensa otro aviador y miembro de la misma promoción, Gonzalo Puigcerver Roma.

En el curso académico 1987-88 ingresó en la Academia General del Aire el Príncipe de Asturias don Felipe de Borbón y Grecia para continuar sus estudios y formación militar que había iniciado dos años antes en la Academia General Militar y en la Escuela Naval de Marín. Su plan 
de formación preveía que en julio de 1989 recibiría su despacho de teniente del Arma de Aviación junto con los equivalentes de los otros dos Ejércitos. En junio de 1988 S.M. El Rey inauguró en el Cuartel General del Ejército del Aire la Cátedra de Pensamiento y Doctrina Aérea Alfredo Kindelán, en un solemne acto en el que estuvo acompañado de diversas autoridades españolas junto con los jefes de Estado Mayor de las Fuerzas Aéreas de la OTAN y los directores de las Escuelas Superiores del Aire o similares de Europa Occidental e Iberoamericanas.

Como consecuencia del histórico referéndum de 1986 por el que se decidió nuestra permanencia en la OTAN, en 1989 comenzaron las negociaciones con España para establecer la forma en la que las Fuerzas Armadas españolas participarían en la defensa común de la Alianza y que se materializaría a través de seis Acuerdos de Coordinación. El primero en el tiempo, y también el más difícil de alcanzar, sería el relativo a establecer la coordinación operativa entre el sistema de Defensa Aérea español y el de la OTAN y cuales serían las áreas geográficas a establecer, así como los procedimientos y los requisitos técnicos. El grupo de trabajo integrado por un representante de cada una de nuestras Fuerzas Armadas fue dirigido por un general del Ejército del Aire y el Acuerdo se firmó en diciembre de 1990 .

\section{El Ejército del Aire en la década de los 90}

El nombramiento del teniente general Ramón Fernández Sequeiros como Jefe de Estado Mayor del Aire al comienzo de los años 90, coincidiría prácticamente en el tiempo con una nueva reorganización del Ejército del Aire, como consecuencia de la estructuración común básica de los tres Ejércitos que había entrado en vigor en 1989 y la creación de los Mandos Operativos. De esta forma el 12 de marzo de 1991, una orden ministerial disponía que el Ejercito del Aire quedaba organizado en un Cuartel General, en el que estaba integrado el Estado Mayor del Aire; la Fuerza Aérea, compuesta por los Mandos Aéreos del Centro, del Estrecho, de Levante y de Canarias y cuyas demarcaciones territoriales coincidían con las Regiones Aéreas Primera, Segunda y Tercera y la Zona Aérea de Canarias respectivamente y el Apoyo Logístico y sus órganos subordinados.

Pero antes, el Ejército del Aire comenzaría a participar en misiones de paz y de ayuda humanitaria, si bien las unidades aéreas de transporte venían desempeñando numerosas misiones y vuelos de socorro prácticamente en todo el mundo con motivos de catástrofes o la aparición de 
hambrunas o plagas en numerosos países. Con ocasión de la Guerra del Golfo y en apoyo de los barcos de la Armada destacados en el Mar Rojo y en el Golfo Pérsico, los aviones de transporte del Ejército del Aire realizaron numerosos vuelos de apoyo a nuestros marinos. Al mismo tiempo, a petición de la Unión Europea Occidental de la que España forma parte, la red hospitalaria militar española ofrecía asistencia médica en previsión de posibles bajas, y el Hospital del Aire reservó durante el curso de las operaciones 150 camas.

A su vez nuestro Hospital en cooperación con el Mando Aéreo de Transporte medicalizó un avión C-130 y preparó hasta nueve equipos médicos instruídos en aerovacuación, de los cuales tres estuvieron destacados en la base aérea francesa de Bricy y alertados para el traslado de heridos, siendo desactivado el destacamento el 1 de marzo de 1991, al finalizar la Operación Tormenta del Desierto. A comienzos de los años 90 el Ejército del Aire se sumaba al proyecto de un Futuro Gran Avión de Transporte Europeo, el FLA.

En 1994 sería designado Jefe del Estado Mayor del Aire el general Ignacio Quintana Arévalo que reemplazaba así a Fernández Sequeiros. Ese año marcaría un hito en la vida del Ejército del Aire pues el 28 de noviembre, partía para la base aérea italiana de Aviano un destacamento del Ejército del Aire compuesto por 8 aviones EF-18 y 2 Hércules C-130H de reabastecimiento en vuelo con los equipos y material necesario, integrado en las fuerzas de la OTAN para participar en misiones de combate a realizar en Bosnia-Hezergovina en apoyo de UNPROFOR. Sería la quinta intervención en guerra de la Aviación española después de la campaña de Marruecos, nuestra Guerra Civil, el frente oriental en la II Guerra Mundial y el conflicto de Ifni-Sáhara. El 25 de mayo de 1995 nuestros pilotos recibieron su bautismo de fuego al volar en una de las misiones de la Operación Deliberated Force. La actuación de nuestros pilotos merecería el reconocimiento internacional y, lo más importante, el de todos los españoles. En septiembre de 1996 fue presentado en Getafe el prototipo español, fabricado por Construcciones Aeronáuticas, del Eurocaza, EF-2000.

La década de los 90 ha sido la de mayor actividad operativa del Ejército del Aire en su corta vida desde su creación en 1939, participando en un gran número de ejercicios aéreos internacionales dentro de las organizaciones supranacionales a las que España pertenece. En ese periodo nuestros cazas EF-18 han cruzado también varias veces el Atlántico Norte para intervenir en territorio norteamericano en los ejercicios Red Flag y nuestro suelo ha sido escenario de diferentes ejercicios aéreos de la OTAN realizados bajo dirección española. Para facilitar la agilidad ope- 
rativa se constituyó el Mando Aéreo de Combate que actúa como Mando Operativo Aéreo.

Relevado el General Quintana por el teniente general Juan Antonio Lombo López, durante el ejercicio de su cargo nuevamente nuestros pilotos tuvieron que intervenir en operaciones de combate al participar en 1999 con la fuerza aérea de la OTAN en una operación destinada a poner fin al conflicto de Kosovo. La Operación denominada Allied Force tendría la característica singular de que por sí solo y por primera vez en la Historia, el Poder Aéreo puso fin a un conflicto militar sin necesidad que tuvieran que participar las fuerzas de superficie.

El nuevo Siglo XXI comenzó con la celebración del 75 Aniversario de los tres primeros Grandes Vuelos de la Aviación Española, los realizados por el Plus Ultra a Buenos Aireś, la Patrulla Elcano a Manila y la Patrulla Atlántida a Guinea Ecuatorial. Junto a estas conmemoraciones ha estado la designación del general Eduardo González-Gallarza Morales como nuevo jefe del Estado Mayor del Aire, dándose con su nombramiento dos felices coincidencias, ser el hijo del héroe del vuelo MadridManila de la Patrulla Elcano y ser el primer y único compañero de Promoción de los tres Ejércitos de S.M. El Rey que ha llegado a ser jefe de Estado Mayor.

Pero la historia del Ejército del Aire estaría incompleta si no se hace mención a las otras actividades no puramente militares que realiza en beneficio de la sociedad y en concreto del pueblo español al que sirve y defiende. Ellas son: extinción de los fuegos forestales, búsqueda y salvamento de aeronaves o barcos accidentados o en situación de emergencia, cooperación con el Servicio de Vigilancia Aduanera en la lucha antidroga, transporte de órganos para transplantes, aeroevacuaciones sanitarias urgentes, vuelos fotográficos y levantamientos cartográficos para organismos estatales y empresas privadas, transporte de personalidades españolas y extranjeras, cooperación con la Aviación Civil facilitándole señales de la red radar de la Defensa Aérea, comprobación y calibración de las ayudas a la Navegación Aérea, reconocimientos médicos a todo el personal aeronáutico de las líneas aéreas y aeroclubes, seguimiento de aves anilladas, estudios aéreos de protección ambiental y colaboraciones diversas con universidades y organismos científicos, bautismos del aire y un largo etcétera. Todo ello sin disminuir en absoluto la misión esencial del Ejército del Aire, la defensa de la Patria y de la vida de los españoles. 